This item was submitted to Loughborough's Research Repository by the author.

Items in Figshare are protected by copyright, with all rights reserved, unless otherwise indicated.

\title{
Expanding impulsive gravitational waves
}

PLEASE CITE THE PUBLISHED VERSION

LICENCE

CC BY-NC-ND 4.0

\section{REPOSITORY RECORD}

Podolsky, J., and J.B. Griffiths. 2019. "Expanding Impulsive Gravitational Waves”. figshare. https://hdl.handle.net/2134/833. 


\title{
Expanding impulsive gravitational waves
}

\author{
J. Podolský* \\ Department of Theoretical Physics, Charles University, \\ V Holešovičkách 2, 18000 Prague 8, Czech Republic. \\ and J. B. Griffiths ${ }^{\dagger}$ \\ Department of Mathematical Sciences, Loughborough University
Loughborough, Leics. LE11 3TU, U.K.
}

June 15, 1999

\begin{abstract}
We explicitly demonstrate that the known solutions for expanding impulsive spherical gravitational waves that have been obtained by a "cut and paste" method may be considered to be impulsive limits of the Robinson-Trautman vacuum type $\mathrm{N}$ solutions. We extend these results to all the generically distinct subclasses of these solutions in Minkowski, de Sitter and anti-de Sitter backgrounds. For these we express the solutions in terms of a continuous metric. Finally, we also extend the class of spherical shock gravitational waves to include a non-zero cosmological constant.
\end{abstract}

Pacs 04.20.Jb, 04.30.Nk

\section{Introduction}

In a classic article in 1972, Penrose [1] presented a novel method for constructing impulsive gravitational waves in a Minkowski background by cutting the space-time along a null hypersurface and then re-attaching the two pieces with a suitable warp. He showed that two types of solution could be constructed by this technique - the familiar impulsive $p p$-waves, and spherical waves that are described as impulsive limits of the RobinsonTrautman waves with spherical wavefronts.

The latter class (and related classes) of spherical impulsive waves have recently received considerable attention. The explicit solution written in a continuous coordinate system has been given by Nutku and Penrose [2] and Hogan [3], [4]. This solution has been interpreted [2] as representing either the snapping of a cosmic string (identified by a deficit angle) in the exterior region generating a spherical impulsive gravitational wave,

${ }^{*}$ E-mail: Podolsky@mbox.troja.mff.cuni.cz

${ }^{\dagger}$ E-mail: J.B.Griffiths@Lboro.ac.uk 
or as having an expanding string generated behind the gravitational wave. The collision (and breaking) of a pair of cosmic strings can also be described in this way.

A solution with a similar interpretation has also been described by Bičák and Schmidt [5]. In this case, however, two null particles recede from a common point generating an impulsive spherical gravitational wave. In this solution, which was obtained as a limiting case of a solution with a boost-rotation symmetry, there is an expanding cosmic string along the axis of symmetry separating the two particles. It was later pointed out [6] that this situation is equivalent to that of the splitting of an infinite cosmic string as described by Gleiser and Pullin [7] or, rather, of two semi-infinite cosmic strings approaching at the speed of light and separating again at the instant at which they collide.

A similar solution in a space-time with a non-vanishing cosmological constant has been given by Hogan [8]. This is a natural extension of the solution [3] to describe a spherical impulsive wave in a de Sitter background, although the solution is also valid when the cosmological constant is negative.

However, despite the statement in Penrose's initial article [1], the above solutions have never been explicitly related to the Robinson-Trautman solutions. Using the original [9] or García-Plebański [10] coordinate systems, it is found that the impulsive limit of the Robinson-Trautman solutions introduces a term in the metric tensor which is quadratic in the Dirac $\delta$-function. The difficulty in treating singular expressions like the square of the $\delta$-distribution can in principle be solved by considering a careful regularization (at least for some special class of regularizing functions) and then performing an appropriate distributional limit of the corresponding well behaved approximation. Such an approach has recently been used, for example, in the context of the equation of geodesic deviation for impulsive pp-waves [11]. Moreover, the recently developed Colombeau theory on nonlinear generalized functions [12], [13] provides a new and rigorous mathematical framework for a possible consistent treatment of such nonlinear operations with distributions. Here, we have not used the full rigour of the Colombeau theory. Nevertheless, we have demonstrated how the problematic singular terms can be removed using an explicit (necessarily discontinuous) coordinate transformation, thus producing a continuous form of the metric.

The first purpose of this paper is to use this transformation to explicitly demonstrate that the impulsive gravitational waves generated by the "cut and paste" method may be considered to be impulsive Robinson-Trautman solutions. This is achieved in section 3.

The second purpose of this paper is to complete the full class of such solutions by taking impulsive limits of all the nine generically distinct subclasses [14] of the RobinsonTrautman type $\mathrm{N}$ vacuum solutions with a cosmological constant. These describe impulsive spherical gravitational waves in de Sitter or anti-de Sitter backgrounds, and are presented in section 4 . The complete family of these solutions is expressed in a coordinate system in which the metric is explicitly continuous. This unifies and generalises previously known results. We will also show that the limits of the distinct classes with different $\epsilon$ (representing all permitted Gaussian curvatures of spacelike surfaces) are locally equivalent. This degeneracy is identical to that observed for the impulsive limits of the Kundt class of solutions as demonstrated recently [15].

Finally, we will also consider the natural extension of the above methods to construct solutions which represent expanding spherical gravitational shock (step) waves. A shock wave of Robinson-Trautman type $\mathrm{N}$ has been constructed by Nutku [16] for the case in which the cosmological constant vanishes. In section 5, we will generalise this to the case of non-zero $\Lambda$ in which the shock wave propagates into a de Sitter or anti-de Sitter background. 


\section{The type N Robinson-Trautman solutions}

Before considering impulsive limits, it is first appropriate to review some general aspects of the type N Robinson-Trautman family of vacuum solutions that will be needed below. These solutions are well known (see [17]). In a familiar coordinate system they can be written in the form

$$
\mathrm{d} s^{2}=2 \frac{r^{2}}{P^{2}} \mathrm{~d} \zeta \mathrm{d} \bar{\zeta}+2 \mathrm{~d} u \mathrm{~d} r-2\left[\epsilon+r(\log P)_{u}-\frac{\Lambda}{6} r^{2}\right] \mathrm{d} u^{2},
$$

where $2 \epsilon(u)$ can be interpreted as the Gaussian curvature of the 2 -surfaces $2 P^{-2} \mathrm{~d} \zeta \mathrm{d} \bar{\zeta}$, and $\Lambda$ is the cosmological constant. In these cases, it is always possible to use the coordinate transformation $u=g(\tilde{u}), r=\tilde{r} / \dot{g}$, where $\dot{g}=\mathrm{d} g / \mathrm{d} \tilde{u}$, to put $\epsilon=-1,0,+1$. It then remains to solve the Robinson-Trautman equation $\Delta \log P=2 \epsilon$, where $\Delta=2 P^{2} \partial_{\zeta} \partial_{\bar{\zeta}}$. A general solution of this equation can be expressed as

$$
P=(1+\epsilon F \bar{F})\left(F_{\zeta} \bar{F}_{\bar{\zeta}}\right)^{-1 / 2},
$$

where $F=F(u, \zeta)$ is an arbitrary complex function of $u$ and $\zeta$, holomorphic in $\zeta$. The only non-vanishing component of the Weyl tensor is given by

$$
\bar{\Psi}_{4}=-\frac{P^{2}}{2 r} F_{\zeta}\left[\frac{1}{F_{\zeta}}\left(\log F_{\zeta}\right)_{u \zeta}\right]_{\zeta} .
$$

It is natural to introduce the invariant classification of the type N Robinson-Trautman vacuum solutions given in [10]. However, we will adopt the slightly modified notation introduced in [14]. This classification is based on two parameters: $\Lambda$ and $\epsilon$, each of which may be either zero, positive or negative. Together, these indicate a total of nine distinct subclasses denoted as $R T N(\Lambda, \epsilon)$.

An alternative representation of this $\operatorname{RTN}(\Lambda, \epsilon)$ class of solutions which is more suitable for interpretation has been given by García Díaz and Plebański [10]. Their metric can be expressed in terms of an arbitrary complex function $f(\xi, u)$ which is holomorphic in the complex spatial coordinate $\xi$ by

$$
\begin{aligned}
\mathrm{d} s^{2}= & 2 v^{2} \mathrm{~d} \xi \mathrm{d} \bar{\xi}+2 \psi \mathrm{d} u \mathrm{~d} v \\
& +2 v \overline{\mathrm{A}} \mathrm{d} \xi \mathrm{d} u+2 v \mathrm{~A} \mathrm{~d} \bar{\xi} \mathrm{d} u+2(\mathrm{~A} \overline{\mathrm{A}}+\psi \mathrm{B}) \mathrm{d} u^{2},
\end{aligned}
$$

where

$$
\begin{aligned}
\mathrm{A} & =\epsilon \xi-v f, \\
\mathrm{~B} & =-\epsilon+\frac{v}{2}\left(f_{\xi}+\bar{f}_{\bar{\xi}}\right)+\frac{\Lambda}{6} v^{2} \psi, \\
\psi & =1+\epsilon \xi \bar{\xi} .
\end{aligned}
$$

The complete family of solutions described by this metric can now be denoted as $R T N(\Lambda, \epsilon)[f]$ in which the free parameters $\Lambda$ and $\epsilon$ and the arbitrary function $f(\xi, u)$ are identified explicitly.

The transformation which relates the above two forms of the Robinson-Trautman solutions is (see [14])

$$
\begin{aligned}
\xi & =F(\zeta, u)=\int f(\xi(\zeta, u), u) \mathrm{d} u \\
v & =\frac{r}{1+\epsilon F \bar{F}} .
\end{aligned}
$$


(If $f$ is independent of $\xi$, it is necessary to put $\xi=F=\zeta+\int f(u) \mathrm{d} u$ ). The Weyl tensor component (using a rescaled tetrad) is now given simply by

$$
\bar{\Psi}_{4}=-\frac{1}{2 r} f_{\xi \xi \xi} .
$$

From this, it is clear that the metric (2) represents only a conformally flat Minkowski or (anti-) de Sitter background when $f$ is no more than quadratic in $\xi$. In all other cases it describes a radiative vacuum space-time of type $\mathrm{N}$ [18].

\section{Impulsive waves in a Minkowski background}

Although the Robinson-Trautman solutions naturally describe "spherical" gravitational waves, it had previously been observed [1], [3] that there are difficulties in considering the impulsive limit in this family since the metric in the standard coordinate system is quadratic in the $\delta$ function, and this gives rise to mathematical problems.

Let us first concentrate on the simplest subclass of type N Robinson-Trautman solutions of the form (2) in which $\Lambda=\epsilon=0$ and consider the impulsive limit as $f(\xi, u) \rightarrow$ $f(\xi) \delta(u)$, i.e. $R T N(\Lambda=0, \epsilon=0)[f=f \delta]$. In this case the line element approaches the form

$$
\begin{aligned}
\mathrm{d} s^{2}= & 2 v^{2} \mathrm{~d} \xi \mathrm{d} \bar{\xi}+2 \mathrm{~d} u \mathrm{~d} v \\
& -2 v^{2} \bar{f} \delta(u) \mathrm{d} \xi \mathrm{d} u-2 v^{2} f \delta(u) \mathrm{d} \bar{\xi} \mathrm{d} u \\
& +\left[2 v^{2} f \bar{f} \delta^{2}(u)+v\left(f_{\xi}+\bar{f}_{\bar{\xi}}\right) \delta(u)\right] \mathrm{d} u^{2} .
\end{aligned}
$$

In this form of the metric, the above difficulty is seen explicitly. The question arises whether a form of the metric exists in which these problematic terms do not appear. In considering a transformation to such a form, it may be observed that, to remove the $\delta$-function components in (4), a discontinuous transformation is necessary. In fact, all the singular components can be removed by making the transformation

$$
\begin{aligned}
& \xi=Z+\Theta(U)\left[-Z+h(Z)+\frac{U}{2 V} \frac{\alpha \bar{\beta}}{\mathcal{A}}\right], \\
& \bar{\xi}=\bar{Z}+\Theta(U)\left[-\bar{Z}+\bar{h}(\bar{Z})+\frac{U}{2 V} \frac{\bar{\alpha} \beta}{\mathcal{A}}\right], \\
& v=V+\Theta(U)\left[-V+V \frac{\mathcal{A}}{|\alpha|}\right], \\
& u=U+\Theta(U)\left[-U+U \frac{|\alpha|}{\mathcal{A}}\right],
\end{aligned}
$$

where $\Theta(U)$ is the Heaviside step function, $h(Z)$ is an arbitrary function related to $f$ by $f(\xi) \equiv h(Z)-Z$ evaluated on $U=0, \alpha=h^{\prime}, \beta=h^{\prime \prime} / h^{\prime}, \mathcal{A}=1-U \beta \bar{\beta} / 4 V$, and the derivative with respect to $Z$ is denoted by a prime. With this, the line element (4) takes the form

$$
\mathrm{d} s^{2}=2 V^{2}\left|\mathrm{~d} Z+\frac{U \Theta(U)}{2 V} \bar{H} \mathrm{~d} \bar{Z}\right|^{2}+2 \mathrm{~d} U \mathrm{~d} V,
$$

where $H=\beta^{\prime}-\frac{1}{2} \beta^{2}=h^{\prime \prime \prime} / h^{\prime}-\frac{3}{2}\left(h^{\prime \prime} / h^{\prime}\right)^{2}$. In this form, the metric is explicitly continuous with the impulsive component $\delta(U)$ appearing only in the curvature tensor. In fact, this is 
the known metric for an expanding impulsive spherical gravitational wave in a Minkowski background obtained by Nutku and Penrose [2] and Hogan [3] using the Penrose [1] "cut and paste" method ${ }^{1}$.

The transformation (5) is an explicit combination of the transformations used in the "cut and paste" method in [3]. It is remarkable that, in this form, it exactly removes all the $\delta$-function components in (6). Conversely, the transformation (5) applied inversely to the Nutku-Penrose-Hogan metric (6) yields the same problematic metric (4) as is obtained as the impulsive limit of the $\operatorname{RTN}(\Lambda=0, \epsilon=0)$ solution. In this sense, the line element (6) may be considered to be equivalent to the impulsive limit of the type $\mathrm{N}$ Robinson-Trautman solution (2) for the case in which $\Lambda=\epsilon=0$.

It may be commented that the discontinuity in the transformation (5) is necessary to remove the impulsive components in the metric (4). This is very similar to the analogous transformation between the Brinkmann and the continuous forms of the impulsive $p p$-wave metric that has recently been investigated elsewhere [19], in which the distributional and continuous forms of the metric have rigorously been shown to be equivalent. However, in the present case, the rigorous calculations are considerable more complicated. This is currently under investigation.

We now move on to describe other spherical impulsive waves in a Minkowski background. These may be considered as belonging to the family $R T N(\Lambda=0, \epsilon= \pm 1)[f=f \delta]$.

In this case, it is first convenient to transform the line element (2) with $f=f(\xi) \delta(u)$ by putting

$$
w=\psi v
$$

With this, (2) takes the form

$$
\begin{aligned}
\mathrm{d} s^{2}= & 2 \frac{w^{2}}{\psi^{2}} \mathrm{~d} \xi \mathrm{d} \bar{\xi}+2 \mathrm{~d} u \mathrm{~d} w-2 \epsilon \mathrm{d} u^{2} \\
& -2 \frac{w^{2}}{\psi^{2}} \bar{f} \delta(u) \mathrm{d} \xi \mathrm{d} u-2 \frac{w^{2}}{\psi^{2}} f \delta(u) \mathrm{d} \bar{\xi} \mathrm{d} u \\
& +\left[2 \frac{w^{2}}{\psi^{2}} f \bar{f} \delta(u)-2 \epsilon \frac{w}{\psi} f \bar{\xi}-2 \epsilon \frac{w}{\psi} \bar{f} \xi+w\left(f_{\xi}+\bar{f}_{\bar{\xi}}\right)\right] \delta(u) \mathrm{d} u^{2} .
\end{aligned}
$$

This clearly includes the above case (4) when $\epsilon=0$, but it also includes the two additional cases when $\epsilon= \pm 1$.

The line element (7) again contains the square of the $\delta$-function, but this problem can similarly be removed by making the more complicated coordinate transformation

$$
\begin{aligned}
\xi & =Z+\Theta(U)\left[-Z+\xi_{p}\right], \\
\bar{\xi} & =\bar{Z}+\Theta(U)\left[-\bar{Z}+\bar{\xi}_{p}\right], \\
w & =V+\Theta(U)\left[-V+w_{p}\right], \\
u & =U+\Theta(U)\left[-U+u_{p}\right],
\end{aligned}
$$

where (assuming $\epsilon \neq 0$ only)

$$
\begin{aligned}
\xi_{p} & =-\frac{\epsilon}{2 \bar{\eta}}\left[(\mathcal{U}-\epsilon \mathcal{V}) \mp \sqrt{(\mathcal{U}-\epsilon \mathcal{V})^{2}+4 \epsilon \eta \bar{\eta}}\right], \\
w_{p} & =-(\mathcal{U}-\epsilon \mathcal{V})-\frac{4 \epsilon \eta \bar{\eta}}{(\mathcal{U}-\epsilon \mathcal{V}) \mp \sqrt{(\mathcal{U}-\epsilon \mathcal{V})^{2}+4 \epsilon \eta \bar{\eta}}},
\end{aligned}
$$

\footnotetext{
${ }^{1}$ In this paper we denote the impulsive wave surface by $U=0$, interchanging the coordinates $U$ and $V$ in [2], [3] and elsewhere.
} 


$$
u_{p}=-\epsilon \mathcal{U}-\frac{2 \eta \bar{\eta}}{(\mathcal{U}-\epsilon \mathcal{V}) \mp \sqrt{(\mathcal{U}-\epsilon \mathcal{V})^{2}+4 \epsilon \eta \bar{\eta}}}
$$

with

$$
\begin{aligned}
\mathcal{U} & =A V-D U, \\
\mathcal{V} & =B V-E U, \\
\eta & =C V-F U,
\end{aligned}
$$

and

$$
\begin{aligned}
& A=\frac{\sqrt{2}}{p\left|h^{\prime}\right|}, \quad B=\frac{|h|^{2}}{\sqrt{2} p\left|h^{\prime}\right|}, \quad C=\frac{h}{p\left|h^{\prime}\right|}, \\
& D=\frac{\sqrt{2}}{\left|h^{\prime}\right|}\left\{\frac{p}{4}\left|\frac{h^{\prime \prime}}{h^{\prime}}\right|^{2}+\epsilon\left[1+\frac{Z}{2} \frac{h^{\prime \prime}}{h^{\prime}}+\frac{\bar{Z} \bar{h}^{\prime \prime}}{2} \overline{h^{\prime}}\right]\right\}, \\
& E=\frac{|h|^{2}}{\sqrt{2}\left|h^{\prime}\right|}\left\{\frac{p}{4}\left|\frac{h^{\prime \prime}}{h^{\prime}}-2 \frac{h^{\prime}}{h}\right|^{2}+\epsilon\left[1+\frac{Z}{2}\left(\frac{h^{\prime \prime}}{h^{\prime}}-2 \frac{h^{\prime}}{h}\right)+\frac{\bar{Z}}{2}\left(\frac{\bar{h}^{\prime \prime}}{\bar{h}^{\prime}}-2 \frac{\bar{h}^{\prime}}{\bar{h}}\right)\right]\right\}, \\
& F=\frac{h}{\left|h^{\prime}\right|}\left\{\frac{p}{4}\left(\frac{h^{\prime \prime}}{h^{\prime}}-2 \frac{h^{\prime}}{h}\right) \frac{\bar{h}^{\prime \prime}}{\bar{h}^{\prime}}+\epsilon\left[1+\frac{Z}{2}\left(\frac{h^{\prime \prime}}{h^{\prime}}-2 \frac{h^{\prime}}{h}\right)+\frac{\bar{Z}}{2} \frac{h^{\prime \prime}}{\bar{h}^{\prime}}\right]\right\},
\end{aligned}
$$

where $f$ is related to $h(Z)$ through the identification $f \equiv \xi_{p}-Z$ evaluated on $U=0$.

With this transformation the line element (7) finally takes the form

$$
\mathrm{d} s^{2}=\frac{2 V^{2}}{p^{2}}\left|\mathrm{~d} Z+\frac{U \Theta(U)}{2 V} p^{2} \bar{H} \mathrm{~d} \bar{Z}\right|^{2}+2 \mathrm{~d} U \mathrm{~d} V-2 \epsilon \mathrm{d} U^{2},
$$

where $p=1+\epsilon Z \bar{Z}$. This is exactly the solution for an expanding spherical impulsive gravitational wave in a Minkowski background that was obtained by Hogan [4]. It is explicitly continuous and obviously reduces to (6) when $\epsilon=0$.

The transformation (8)-(11) is evidently rather complicated. However, it reduces to an identity when $U<0$ so that the metric (7) with $f=0$ goes directly to (12). On the other hand, for $U>0$ the transformation (8)-(9) is inverse to

$$
\begin{aligned}
\mathcal{U} & =\frac{w}{\psi}-\epsilon u, \\
\mathcal{V} & =\frac{\xi \bar{\xi} w}{\psi}-u, \\
\eta & =\frac{\xi w}{\psi},
\end{aligned}
$$

and brings the same "background" $R T N(\Lambda=0, \epsilon)[f=0]$ metric given by $(7)$ to an explicit Minkowski form

$$
\mathrm{d} s^{2}=2 \mathrm{~d} \eta \mathrm{d} \bar{\eta}-2 \mathrm{~d} \mathcal{U} \mathrm{d} \mathcal{V} .
$$

This metric is related to (12) with $U>0$ by (10)-(11). Note that the transformation (8)(11) cannot simply be applied when $\epsilon=0$. The reason is that (9) is not a correct inverse relation to (13) in this case. However, using the correct one $\xi=\eta / \mathcal{U}, v=\mathcal{U}, u=\eta \bar{\eta} / \mathcal{U}-\mathcal{V}$ with (10)-(11), we get exactly the transformation (5) after a trivial rescaling $\xi \rightarrow \sqrt{2} \xi$, $v \rightarrow v / \sqrt{2}$ and $u \rightarrow \sqrt{2} u$. 
Let us here also present some useful relations valid for the above transformations. First, it can easily be shown that $\sqrt{(\mathcal{U}-\epsilon \mathcal{V})^{2}+4 \epsilon \eta \bar{\eta}}=|w|$, so that we have to consider only the upper signs in Eqs. (9) when $w>0$ and the lower signs when $w<0$. This naturally explains an apparent ambiguity of the transformation.

Secondly, it immediately follows from Eqs. (13) that $\eta \bar{\eta}-\mathcal{U V} \equiv u(w-\epsilon u)$. Substituting from (10) and using the identities

$$
\begin{aligned}
C \bar{C}-A B & =0, \\
F \bar{F}-D E & =-\epsilon, \\
A E+B D-C \bar{F}-\bar{C} F & =1,
\end{aligned}
$$

we get an interesting relation

$$
u(w-\epsilon u)=U(V-\epsilon U),
$$

which will be important in the next section.

Note finally that in the Robinson-Trautman metrics with general profile function $f(\xi, u)$, the 2-surfaces spanned by $\zeta$ have constant curvature which may be zero, positive or negative according to the value of $\epsilon$. However, it can be shown that, for the limiting case of impulsive waves, these three cases are equivalent. Indeed, the simple coordinate transformation

$$
\begin{aligned}
\xi & \rightarrow \xi \frac{v}{v-\epsilon u}, \\
v & \rightarrow v-\epsilon u \\
u & \rightarrow u \frac{\psi v-\epsilon u}{v-\epsilon u}
\end{aligned}
$$

takes the solution (4) $R T N(\Lambda=0, \epsilon=0)[f=f \delta]$ to $(7) R T N(\Lambda=0, \epsilon)[f=f \delta]$. This degeneracy of the impulsive limit of the Robinson-Trautman solutions is similar to that of the Kundt class of solutions for non-expanding impulsive waves as shown in [15].

\section{Impulsive waves in a de Sitter or anti-de Sitter background}

It is fairly straightforward to generalise the above solution (6) to the case of an expanding spherical gravitational wave in a de Sitter, or anti-de Sitter background. This was achieved by Hogan [8] expressing the metric in the similar continuous form:

$$
\mathrm{d} s^{2}=\frac{1}{\left(1+\frac{\Lambda}{6} U V\right)^{2}}\left[2 V^{2}\left|\mathrm{~d} Z+\frac{U \Theta(U)}{2 V} \bar{H} \mathrm{~d} \bar{Z}\right|^{2}+2 \mathrm{~d} U \mathrm{~d} V\right] .
$$

By applying the transformation (5), we can relate (16) exactly to the metric (4) multiplied by a conformal factor $\left(1+\frac{\Lambda}{6} u v\right)^{-2}$ since (5) has the nice property that $u v=U V$. Performing the further transformation

$$
\tilde{v}=\frac{v}{1+\frac{\Lambda}{6} u v}
$$

and dropping the tildes, we immediately obtain the impulsive subclass of the metric (2) which represents the class of solutions denoted as $\operatorname{RTN}(\Lambda, \epsilon=0)[f=f \delta]$ in which $\Lambda$ may be zero, positive or negative. 
It is now natural to look for the alternative form of this class of solutions for which both $\Lambda$ and $\epsilon$ may be non-zero. This corresponds to a different coordinate representation of (16) similar to the alternative form (12) of the solution (6) for the case when $\Lambda=0$. A general solution satisfying these properties would then be valid for all possible values of $\Lambda$ and $\epsilon$ thus covering all nine generically different subclasses of the Robinson-Trautman vacuum type $\mathrm{N}$ solutions.

This general form of the metric in a continuous coordinate system may be written in the form

$$
\mathrm{d} s^{2}=\frac{1}{\left[1+\frac{\Lambda}{6} U(V-\epsilon U)\right]^{2}}\left[\frac{2 V^{2}}{p^{2}}\left|\mathrm{~d} Z+\frac{U \Theta(U)}{2 V} p^{2} \bar{H} \mathrm{~d} \bar{Z}\right|^{2}+2 \mathrm{~d} U \mathrm{~d} V-2 \epsilon \mathrm{d} U^{2}\right] .
$$

As in the above case, this can be derived using the transformation (8)-(11) from the metric (7) multiplied by the conformal factor

$$
\frac{1}{\left[1+\frac{\Lambda}{6} u(w-\epsilon u)\right]^{2}}
$$

considering the identity (14). Further, this can be written in the form of the most general impulsive metric of the Robinson-Trautman type

$$
\begin{aligned}
\mathrm{d} s^{2}= & 2 \frac{w^{2}}{\psi^{2}} \mathrm{~d} \xi \mathrm{d} \bar{\xi}+2 \mathrm{~d} u \mathrm{~d} w+\left(\frac{\Lambda}{3} w^{2}-2 \epsilon\right) \mathrm{d} u^{2} \\
& -2 \frac{w^{2}}{\psi^{2}} \bar{f} \delta(u) \mathrm{d} \xi \mathrm{d} u-2 \frac{w^{2}}{\psi^{2}} f \delta(u) \mathrm{d} \bar{\xi} \mathrm{d} u \\
& +\left[2 \frac{w^{2}}{\psi^{2}} f \bar{f} \delta(u)-2 \epsilon \frac{w}{\psi} f \bar{\xi}-2 \epsilon \frac{w}{\psi} \bar{f} \xi+w\left(f_{\xi}+\bar{f}_{\bar{\xi}}\right)\right] \delta(u) \mathrm{d} u^{2}
\end{aligned}
$$

by putting

$$
\begin{aligned}
\tilde{w} & =\frac{w}{1+\frac{\Lambda}{6} u(w-\epsilon u)}, \\
\tilde{u} & =\int \frac{\mathrm{d} u}{1-\epsilon \frac{\Lambda}{6} u^{2}},
\end{aligned}
$$

and dropping the tildes. The metric (20) describes all possible subclasses $R T N(\Lambda, \epsilon)[f=$ $f \delta]$ in which $\Lambda$ and $\epsilon$ may be zero, positive or negative. It reduces to the forms (7) when $\Lambda=0$ and further to (4) when $\epsilon=0$.

The above argument demonstrates that the continuous form of the metric given in (18) covers all possible subclasses. In terms of continuous coordinates, the new metric (18) clearly reduces to the three previously known forms (12), (16) and (6) when $\Lambda=0$ or $\epsilon=0$ or both.

However, it should again be stressed that in the impulsive cases for each $\Lambda$, the forms for different $\epsilon$ are equivalent. This can easily be shown by rewriting the impulsive subclasses $R T N(\Lambda, \epsilon=0)[f=f \delta]$ of the metric (2) using the transformation (17) in the form which is conformal to (4) with the conformal factor $\left(1+\frac{\Lambda}{6} u v\right)^{-2}$. Now the transformation (15) brings the metric to the form (7) of $R T N(\Lambda=0, \epsilon)[f=f \delta]$ multiplied by the conformal factor $\left[1+\frac{\Lambda}{6} u(w-\epsilon u)\right]^{-2}$. Finally, using (21) we obtain $(20)$, i.e. $R T N(\Lambda, \epsilon)[f=f \delta]$. 


\section{Shock waves in de Sitter and anti-de Sitter back- grounds}

It can be observed that the above solutions describing an impulsive gravitational wave can easily be adapted to describe shock (step) waves. Spherical shock waves belonging to the Robinson-Trautman type N class have been explicitly constructed by Nutku [16]. These cover the case in which the cosmological constant vanishes so that the shock propagates into a Minkowski background. This was obtained initially using a modification of Penrose's method of identification with warp [1] in which the space-time behind the wavefront is not flat. When the metric functions are smooth, these solutions are equivalent to the Robinson-Trautman vacuum solutions.

It may be noted that there are also other solutions for shock waves with a spherical wave-front which propagate into a Minkowski background [20]. These are algebraically general, indicating the presence of backscattering (wave tails). However, here we will con-

centrate on the Robinson-Trautman solutions which take a similar form to the impulsive solutions described above. In fact we can easily extend the known solution to the case in which the cosmological constant is non-zero.

We may again start with the metric (2) which describes the Robinson-Trautman type $\mathrm{N}$ vacuum solutions in the García-Plebański coordinate system [10]. Using the transformation

$$
\begin{aligned}
\xi & =F(\zeta, u) \\
v & =\frac{\tilde{r}}{\sqrt{F_{\zeta} \bar{F}_{\bar{\zeta}}}}
\end{aligned}
$$

we obtain the Nutku form of the metric

$$
\begin{aligned}
\mathrm{d} s^{2}= & 2 \tilde{r}^{2} \mathrm{~d} \zeta \mathrm{d} \bar{\zeta}+2 P \mathrm{~d} u \mathrm{~d} \tilde{r} \\
& +2 \tilde{r} P_{\zeta} \mathrm{d} \zeta \mathrm{d} u+2 \tilde{r} P_{\bar{\zeta}} \mathrm{d} \bar{\zeta} \mathrm{d} u+\left(\frac{\Lambda}{3} \tilde{r}^{2} P^{2}-2 \epsilon\right) \mathrm{d} u^{2},
\end{aligned}
$$

where $P(\zeta, \bar{\zeta}, u)$ is given by $(1)$ in terms of $F(\zeta, u)$ which satisfies

$$
\frac{\partial F}{\partial u}=f(\xi=F(\zeta, u), u)
$$

where $f$ is the arbitrary complex function which appears in the metric (2).

The spherical shock wave arises when the function $F(\zeta, u)$ is of the form

$$
F=F(\zeta+u \Theta(u))
$$

With this, the metric (22) generalises the Nutku solution [16] to the case of non-vanishing cosmological constant.

In view of (3), the simplest spherical shock wave in Minkowski, de Sitter and antide Sitter backgrounds is generated by the function $f$ which is cubic in $\xi$. It is natural to consider

$$
f(\xi, u)=-\Theta(u) \xi^{3}
$$

which corresponds to

$$
F(\zeta, u)=\frac{1}{\sqrt{2} \sqrt{\zeta+u \Theta(u)}}
$$




$$
P=\sqrt{2|\zeta+u \Theta(u)|}(2|\zeta+u \Theta(u)|+\epsilon) .
$$

Although the metric (2) is discontinuous in this case, the metric (22) is explicitly continuous and there is a discontinuity in the Weyl tensor component (3).

\section{Conclusions}

By constructing explicit transformations, we have shown that the known solutions for spherical impulsive gravitational waves constructed by the "cut and paste" method may be considered to be impulsive limits of the type N Robinson-Trautman family of solutions.

We have considered these impulsive limits for all the nine generically different subclasses of the Robinson-Trautman type N solutions $\operatorname{RTN}(\Lambda, \epsilon)$ and presented a continuous coordinate system unifying and generalizing previously known forms for arbitrary $\Lambda$ and $\epsilon$. However, as in the non-expanding type N Kundt class, the solutions for different values for $\epsilon$ are found to be equivalent in these impulsive limits.

Finally, we have presented a family of exact solutions for expanding spherical shock waves of Robinson-Trautman type in conformally flat backgrounds. This generalises a previously known solution in a Minkowski background to include the equivalent solution in de Sitter or anti-de Sitter backgrounds.

\section{Acknowledgments}

This work was supported by a visiting fellowship from the Royal Society and, in part, by the grant GACR-202/99/0261 of the Czech Republic.

\section{References}

[1] Penrose R 1972 General Relativity ed L O’Raifeartaigh (Oxford: Clarendon)

[2] Nutku Y and Penrose R 1992 Twistor Newsletter No. 34, 11 May, 9

[3] Hogan P A 1993 Phys. Rev. Lett. 70117

[4] Hogan P A 1994 Phys. Rev. D 496521

[5] Bičák J and Schmidt B 1989 Class. Quantum Grav. 61547

[6] Bičák J 1990 Astron. Nachr. 311189

[7] Gleiser R and Pullin J 1989 Class. Quantum Grav. 6 L141

[8] Hogan P A 1992 Phys. Lett. A 171, 21

[9] Robinson I and Trautman A 1960 Phys. Rev. Lett. 4431

[10] García Díaz A and Plebański J F 1981 J. Math. Phys. 222655

[11] Steinbauer R 1998 J. Math. Phys. 392201

[12] Colombeau J F 1984 New Generalized Functions and Multiplication of Distributions (Amsterdam: North Holland) 
[13] Colombeau J F 1992 Multiplication of Distributions (Berlin: Springer)

[14] Bičák J and Podolský J submitted to J. Math. Phys.

[15] Podolský J 1998 Class. Quantum Grav. 153229

[16] Nutku Y 1991 Phys. Rev. D 443164

[17] Kramer D, Stephani H, MacCallum M A H and Herlt E 1980 Exact Solutions of Einstein's Field Equations (Cambridge: Cambridge University Press)

[18] Bičák J and Podolský J submitted to J. Math. Phys.

[19] Kunzinger M and Steinbauer R 1999 Class. Quantum Grav. 161255

[20] Alekseev G A and Griffiths J B 1996 Class. Quantum Grav. 13 L13 